\title{
Effect of additive noise on phase measurement in digital holographic microscopy
}

\author{
Nitesh Pandey • Bryan Hennelly
}

Received: 29August 2010 / Revised: 29 September 2010 / Accepted: 13 October 2010

(C) 3D Research Center and Springer 2011

\begin{abstract}
Digital holographic microscopy is a quantitative phase measurement technique that can provide nanometer resolution of the thickness or surface profile of an object. We analyze the influence of additive noise in the hologram plane on the accuracy of phase measurement. We analyze Gaussian distributed and Poisson distributed shot noise in the camera plane and we develop a model for quantifying the phase error in the reconstructed phase.
\end{abstract}

Keywords: Noise, Phase Measurement, and Digital Holographic Microscopy

\section{Introduction}

Digital holographic microscopy (DHM) is a powerful method for single-shot quantitative phase contrast microscopy. It is highly applicable in imaging biological specimens in a non-invasive manner ${ }^{1-3}$ and in surface profile measurement ${ }^{4}$. Using a dual wavelength technique, it has been shown that sub-nanometer accuracy can be achieved ${ }^{5}$. An important advantage of DHM is that the aberrations resulting from imperfect optics can be completely compensated $^{6,7}$. The digital holographic imaging system is a hybrid opto-digital system in which the wavefield from an object is captured on an image sensor by interference and processed numerically to extract the phase of the wavefield. This is susceptible to various sources of noise. Sources of noise in holographic imaging include quantization noise, shot noise, thermal noise, vibrations and sometimes even speckle. At low exposures, the prevalent and the limiting noise is shot noise ${ }^{8}$. For many applications, it is of interest to perform digital holography at low exposures. In biology, for the visualization of fast surface changes of cells and in metrology for vibration measurement, a fast frame rate is

Nitesh Pandey ${ }^{1} \cdot$ Bryan Hennelly $^{1}$

${ }^{1}$ NUI Maynooth, Maynooth, Kildare IRELAND

e-mail: nitesh.pandey@gmail.com needed. In Ref. 9, the authors presented a method based on heterodyne holography where they have demonstrated recording and reconstructing images at extremely low signal levels. Yamamoto et.al have recently demonstrated the possibility of object reconstruction under very weak illumination (43 photons per second $)^{10}$. In such cases, the issue of shot noise is particularly important.

The influence of shot noise in DHM was first studied by Charrière et $\mathrm{al}^{11,12}$. They have experimentally investigated the influence of the parameters like the gain of the CCD, power in the recording beams and the beam ratio on the standard deviation in the phase error. They also showed methods of reducing shot noise by combining multiple exposures. In Ref. 11, the authors used a SNR metric based on statistical decision theory, which is suitable for biological microscopy specimens, to study the influence of recording parameters on the reconstructed holograms in presence of shot noise.

In this paper, we attempt to provide a statistical measure of the phase error in (DHM). In section 2, we show that a noise source in the hologram plane results in a complex noise in the reconstruction plane for off-axis Fresnel DHM. We show that both the Fourier filtering and the Fresnel propagation lead to the Gaussian statistics in the reconstruction plane. This complex noise is shown to have real and imaginary parts which are Gaussian distributions, the variance of which is related to the variance of the original noise source in the hologram plane. In section 2.3, we proceed to show how we can develop this concept further to develop a theoretical model to predict the phase error in DHM resulting from a noise source in the hologram plane. In section 3.1 we focus on the case where the original noise source in the hologram plane is described by a Gaussian noise. Such noise sources include thermal noise. In section 3.2, we focus on the case where the original noise source in the hologram plane is generated by a Poisson process and finally give a brief conclusion. 


\section{Noise in digital holography}

In DHM, noise occurs in the capture plane at the image sensor. This is always real in nature since it occurs due to the square law detection. The hologram is then reconstructed to extract the phase information of the object. The reconstruction in DHM is generally obtained by firstly filtering the hologram in the Fourier domain to remove the twin image and zero order terms and secondly by computing the Fresnel transform. This gives rise to a second type of noise in the reconstruction plane. Here we show that both the filtering process and the propagation process tend to make the real noise in the hologram plane as a complex Gaussian noise. For our study we use a hologram acquired from a commercial Digital holographic microscope (Lyncee-Tec DHM-T1000). This has transmission off-axis Fresnel architecture. The object we used is a micro-lens array. The hologram is captured under good recording conditions with an exposure time of $314 \mu \mathrm{s}$. The wavelength used in this system is $\lambda=682 \mathrm{~nm}$ and the pixel pitch is $6.45 \mu \mathrm{m}$. The hologram has a size of $1392 \times 1040$ pixels. We reconstruct a region of $1024 \times 1024$ pixels from the original hologram and the size of the mask is $450 \times 450$ pixels in the Fourier domain. Fig. 1(a) shows the hologram. Fig. 1(b) shows the DFT before application of the filtering mask. Fig. 1(c) shows the amplitude in the reconstruction and Fig. 1(d) shows the phase of the microlenses structure.

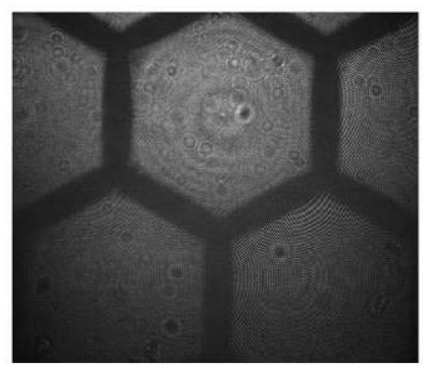

(a) Hologram

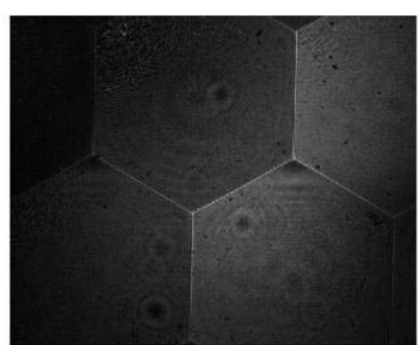

(c) Amplitude of reconstruction

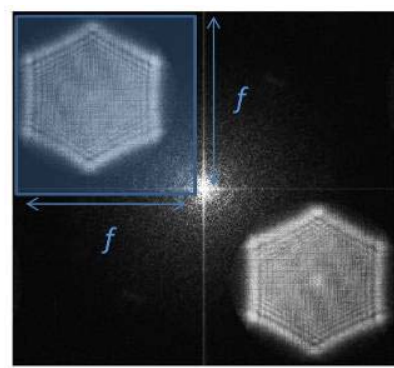

(b) Fourier plane

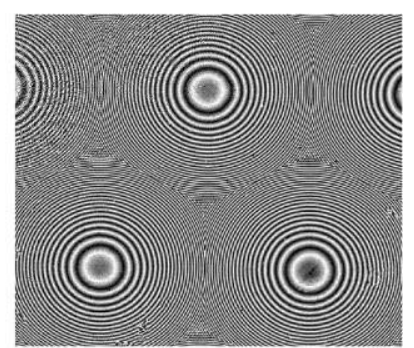

(d) Phase

of reconstruction
Fig. 1 (a) Hologram of phase object, (b) Fourier transform showing the filtered area. (c) Amplitude of the reconstruction and (d) Phase of the reconstruction.

\subsection{Fourier filtering}

Fourier filtering is used to remove the zero-order term and the twin image in the hologram. This is accomplished by multiplying with a binary mask in the Fourier plane which has a passband over the object region. The filtering process in the Fourier plane can be viewed as a convolution with a complex $\operatorname{sinc}(\mathrm{x})$ function or a Bessel function in the case of a circularly shaped filter. As we filter the hologram, the nature of the noise transforms from uniform real to complex Gaussian. To examine this transformation, we consider real uniformly distributed zero mean random white noise in the hologram plane and study the characteristics of the resulting complex filtered noise. Quantization noise is an example of such a uniformly distributed zero-mean white noise. We use kurtosis as a measure of the 'Gaussianness' of the distribution of the real and imaginary parts ${ }^{13}$.

The kurtosis of a variable $\mathrm{x}$ with mean $\mu$ and standard deviation $\sigma$ is defined by

$$
\text { Kurt }=\frac{E\left[(x-\mu)^{4}\right]}{\sigma^{4}}-3
$$

Uniformly distributed variables have a kurtosis of $-6 / 5$ while perfectly Gaussian variables have a kurtosis of 0 . We perform Fourier filtering on this noise for different filter sizes and calculate the kurtosis of the real and imaginary parts of the resulting complex noise as a function of the filter size.

In Fig. 2 it can be seen that the kurtosis of the real part of the noise decreases from 0 to -1.2 as the filter size increases. While the kurtosis of the imaginary part stays almost constant. This is expected as the noise in the hologram plane is real and transitions to being complex when sufficient frequencies are removed. In the case of Fresnel off-axis or Fresnel-infocus digital holography, the filter size in the Fourier plane in number of pixels is at least one half of the number of pixels in the hologram. For this filter size, the noise is complex Gaussian with both real and imaginary points having the same variance.

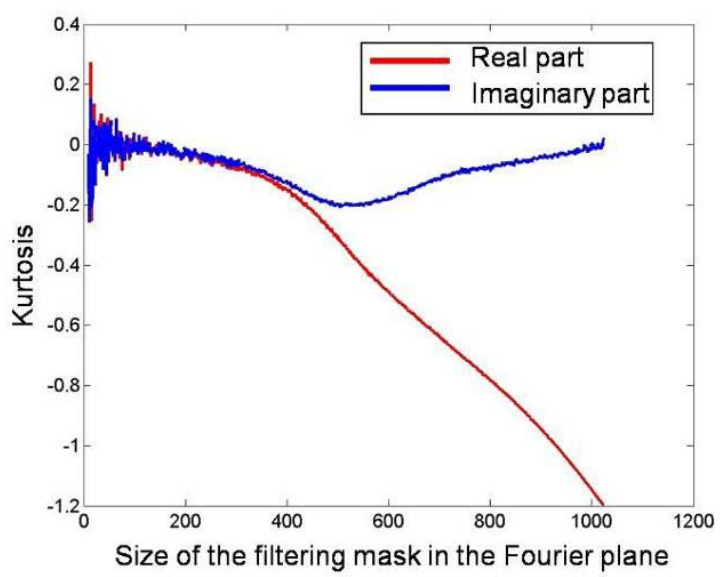

Fig. 2 Kurtosis of the real and imaginary part of the complex noise resulting from Fourier filtering.

\subsection{Numerical Propagation}

The next step in the reconstruction algorithm is numerical propagation of the retrieved complex waveforms by simulating the Fresnel transform. In general the Fresnel transform is implemented by using the convolution approach in which the discrete Fourier transform (DFT) of the filtered hologram is multiplied by a discrete quadratic phase function and then an inverse DFT is performed ${ }^{14,15}$ This further contributes to the final distribution of the Gaussian noise in the reconstruction plane. This is demonstrated using the equations below for a simple 1D 
case. Let $H(n)$ be the filtered hologram and $D$ represent its DFT.

$$
\begin{aligned}
& D(k)=\frac{1}{\sqrt{N}} \sum_{n=0}^{N-1} H(n) \exp \left(-i \frac{2 \pi k n}{N}\right) \\
& =\frac{1}{\sqrt{N}} \sum_{n=0}^{N-1} H(n) \cos \left(\frac{2 \pi k n}{N}\right)-\frac{1}{\sqrt{N}} \sum_{n=0}^{N-1} H(n) \sin \left(\frac{2 \pi k n}{N}\right)
\end{aligned}
$$

Let $C$ represent the discrete quadratic phase factor for a wavelength $\lambda$ and distance $d$.

$$
C(n)=\exp \left(i \pi \frac{n^{2}}{\lambda d}\right)=\cos \left(\frac{\pi n^{2}}{\lambda d}\right)+i \sin \left(\frac{\pi n^{2}}{\lambda d}\right)
$$

where $n=n^{\prime} \Delta x$ for $n^{\prime}=N / 2$ to $n^{\prime}=N / 2-1$ where $\Delta \mathrm{x}$ is the pixel pitch of the camera.

The final reconstruction of this is given by

$$
O(k)=\operatorname{IDFT}[D(n) C(n)]
$$

thus giving

$$
O(k)=\frac{1}{\sqrt{N}} \sum_{n=0}^{N-1} D(n) C(n) \exp \left(i \frac{2 \pi k n}{N}\right)
$$

where IDFT is the inverse discrete Fourier transform. For ease of notation, we have ignored some constant phase factors. It can be seen that each value in the final reconstruction $O$ is the result of a weighted sum of the values of the previous DFT. Since the filtering process and the numerical Fresnel transform are both linear and since we assume the noise to be additive, we can say that noise is also 'propagated' using the same algorithm and each value of the noise in the output plane results from the sum of a large number of random values taken from the input plane. The noise term at each point in the reconstruction plane is therefore given by a large sum of randomly weighted phasors, the angles of which are uniformly distributed between $-\pi$ and $\pi$.

We can use the central limit theorem to conclude that the noise in the reconstruction plane after Fourier filtering and Fresnel propagation has Gaussian statistics. The statistical properties of the complex noise are similar to that of speckle $^{16}$. The real and imaginary parts are Gaussian distributed while the amplitude is Rayleigh distributed and the phase of the noise is uniformly distributed in the range $[-\pi, \pi]$.

Let $\sigma_{I}^{2}$ represents the variances of the real additive noise in the input plane and $\sigma^{2} O_{\mathrm{Re}}, \sigma^{2} O_{\mathrm{Im}}$ represent the variances of the real and imaginary parts of the resulting noise at the output plane respectively. By using Parseval's theorem, we can say

$$
\sigma^{2} O_{\mathrm{Re}}+\sigma^{2} O_{\mathrm{Im}}=\sigma_{I}^{2}
$$

Assuming the energy to be evenly distributed between the real and imaginary parts we can assume

$$
\sigma^{2} O_{\mathrm{Re}}=\sigma^{2} O_{\mathrm{Im}}=\frac{1}{2} \sigma^{2}{ }_{I}
$$

We assume that the noise energy is evenly distributed throughout the frequency domain. If there is a bandpass filter in the frequency domain with a passband of $f^{2}$. i.e. the spatial frequency bandwidth of the transmitting mask in the DFT plane is $f$ times the bandwidth of the hologram in both dimensions, then by once again using Parseval's theorem, we can conclude that the final variance becomes $f^{2} \sigma^{2}$.

Thus the real and imaginary parts of the noise in the reconstruction plane have Gaussian distributions both with a variance given by $f^{2} \sigma^{2}{ }_{I} / 2$.

\subsection{Statistics of the error in phase}

If we consider the reconstructed signal, $S_{e}$ to be given by the sum of the noise free signal, $S_{o}$ and the error inducing noise term, $E$. i.e $S_{e}=S_{o}+E$. Therefore the phase error can be defined as the difference between the phase of the original noise free signal $\left(\angle S_{o}\right)$ and the phase of the noisy signal $\left(\angle S_{e}\right)$;

$\theta_{\text {err }}=\angle S_{e}-\angle S_{o}$

Letting the reconstruction $S_{o}=A \exp \left(i \angle S_{o}\right)$, where $A$ is the uniform amplitude in the reconstruction. Since we are subtracting the phase of So from Se to find the phase error, $\theta_{\text {err }}$ can be written as

$\theta_{\text {err }}=\angle\left[\mathrm{S}_{\mathrm{e}} \exp (\mathrm{i} \angle \mathrm{So})\right]$

since $S_{e}=S_{o}+E$, we can write this as

$\theta_{\text {err }}=\angle\left[\left(\mathrm{S}_{\mathrm{o}}+\mathrm{E}\right) \exp \left(\mathrm{i} \angle \mathrm{S}_{\mathrm{o}}\right)\right]=$

$\angle\left[\mathrm{S}_{\mathrm{o}} \exp \left(\mathrm{i} \angle \mathrm{S}_{\mathrm{o}}\right)+\mathrm{E} \exp \left(\mathrm{i} \angle \mathrm{S}_{\mathrm{o}}\right)\right]=\angle\left[\mathrm{A}+\mathrm{E} \exp \left(\mathrm{i} \angle \mathrm{S}_{\mathrm{o}}\right)\right]$

This shows that the phase error in the reconstruction plane has the same form as that of the phase distribution in the sum of a uniform background of amplitude, $A$ and a speckle pattern. We restate here that our assumption that the object has uniform amplitude in the reconstruction, i.e $A$ is uniform. Such an assumption is valid for phase only objects. In this case the probability distribution of the phase error, $\theta_{\text {err }}$ can be interpreted as the distribution of phase in the sum of a constant phasor and a random speckle like noise. This is similar to the phase distribution in the coherent sum of a uniform background with the speckle field emitting from a rough surface. The probability distribution of the phase in such a sum has been shown ${ }^{16,17}$ to be

$$
p\left(\theta_{e r r}\right)=\frac{1}{2 \pi} \exp \left(\frac{-A^{2}}{2 \sigma^{2}}\right)\left\{\begin{array}{l}
1+\frac{A}{\sigma_{R}} \sqrt{2 \pi} \cos \left(\theta_{\text {err }}\right) \\
\exp \left(\frac{A^{2} \cos ^{2} \theta_{\text {err }}}{2 \sigma_{R}{ }^{2}}\right)\left[1-Q\left(\frac{A \cos \left(\theta_{e r r}\right)}{\sigma_{R}}\right)\right]
\end{array}\right\}
$$

where $\sigma_{R}$ is the standard deviation of the real part(or imaginary part) of the complex noise in the reconstruction plane. From our previous analysis in section 2.1, this is equal to $\sigma_{R}=\mathrm{f} \sigma_{I} / \sqrt{2}$ where $\sigma_{I}$ is equal to the standard deviation of the noise in the input (camera) plane and

$$
Q(x)=\frac{1}{2 \pi} \int_{x}^{\infty} \exp \left(\frac{-x^{2}}{2}\right) d x
$$

Equation 11 is also used to model the noise in MRI data ${ }^{18}$ and in the general case of the phase error when Gaussian noise is added to a complex sinusoidal signal ${ }^{19}$.

When $A \gg>\sigma_{R}$, Equation 11 can be approximated as a Gaussian of the form

$$
p\left(\theta_{e r r}\right)=\frac{A}{\sqrt{2 \pi} \sigma_{R}} \exp \left(\frac{-\theta_{e r r}{ }^{2} A^{2}}{2 \sigma_{R}{ }^{2}}\right)
$$

with a standard deviation of

$$
\sigma_{\theta \text { err }}=\frac{\sigma_{R}}{A}=\frac{f \sigma_{I}}{A \sqrt{2}}
$$




\section{Additive noise in the Hologram plane}

\subsection{Application to additive white Gaussian noise}

Gaussian noise is a general model that is often used in digital signal processing to describe the noise from various sources. The combined effect of all the noises due to quantization, thermal noise, shot noise and readout noise can be described as a zero mean additive white Gaussian noise (AWGN). We numerically generate AWGN with different standard deviations and add them to our sample hologram. The noisy holograms are reconstructed using the convolution method for simulating the Fresnel transform. The phase of the reconstruction is calculated and the standard deviation in the phase error is calculated by least squares fitting the observed distribution to a nonnormalized Gaussian. This value is compared with the predicted value given by Equation 14. Any aberrations resulting from the setup are suppressed by capturing the interference patterns when there is no object and reconstructing it. The phase of this reconstruction is then subtracted from the reconstruction of the object. Our acquired holograms has some noise like microscopic dust particles, spurious reflections and other noise sources like readout noise, quantization noise etc but since we are measuring the error in phase upon introducing computer generated noise, the hologram we acquire is treated as a benchmark noise free hologram and we compare the noisy reconstructions against this hologram.

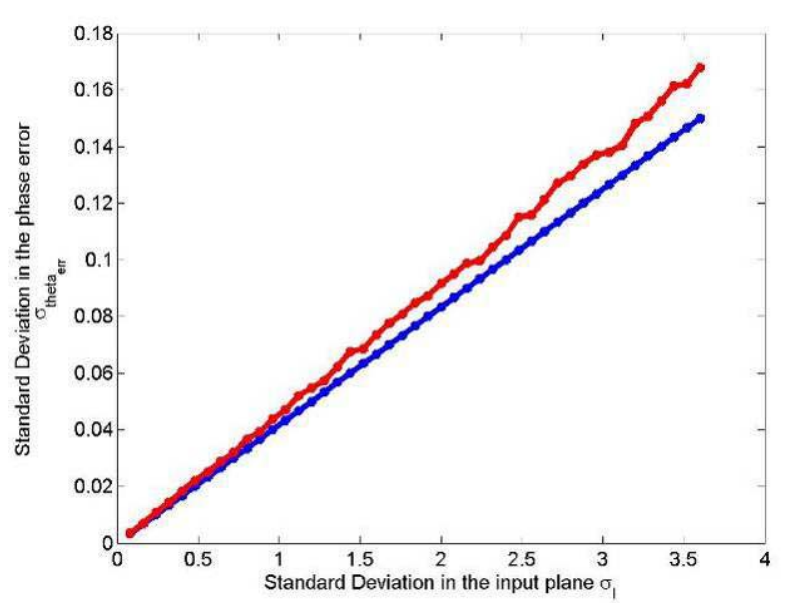

Fig. 3 Standard deviation of the phase error vs. the standard deviation in the camera plane of the hologram for Gaussian noise.

Fig. 3 shows the standard deviation in the phase error vs. the standard deviation of the noise in the input plane for both small errors. We see an approximately linear relationship. The phase error starts deviating when the noise standard deviation increases beyond a certain extent and this is because of the departure from the assumption that $A \gg \sigma_{R}$. The standard deviation of the original hologram pixel values is 11.9 and at a relatively high additive noise of $\sigma_{I}=3.5$, the deviation in the predicted and actual values is $12 \%$. In the case of even high noise, the noise in the capture plane dominates the phase signal. The phase of the reconstructed signal is immersed in noise and the values tend to become uniformly distributed in $[-\pi, \pi]$. This can be seen in Equation 11. In the limit of $A / \sigma_{R}$ going to zero, the phase error signal becomes uniformly distributed with a probability density of $1 / 2 \pi$ between $[-\pi, \pi]$ and thus with a variance of $\pi^{2} / 3$. Thus the standard deviation in the measured error becomes $\pi / \sqrt{3}$.

At low exposures, the influence of noises like thermal noise and quantization noise can be reduced by cooling the CCD chip and by using a higher bit rate camera respectively. The ultimate noise limitation is due to the fundamental shot noise at low light imaging.

\subsection{Application to Poisson noise}

In this subsection, we investigate the phase error in DHM when the noise in the hologram plane is generated from a Poisson distribution.

Our theoretical model complements the experimental investigations done in ${ }^{11,12}$. Poisson noise is a data dependent noise which occurs due to the random and discrete nature of the photons and photoelectrons involved in the digital image formation process. It can be treated as additive in nature ${ }^{20}$. This noise is a fundamental limitation to imaging ${ }^{8}$ and is particularly influential at low lighting conditions.

Since the amount of shot noise depends only on the signal and not on any other external factors, we can provide an estimate for the phase error based on the power of the signal itself. We can estimate the standard deviation of the shot noise in the following way. An important characteristic of a Poisson distributed variable is that the variance is equal to the mean. The variance of Poisson noise is equal to the mean of the signal $\mu$. Thus the standard deviation is given by $\sqrt{\mu}$. When this hologram is amplified by a factor $\alpha$, the amplitude in the reconstruction becomes $\alpha A$ and the mean becomes $\alpha \mu$. Once again A represents the amplitude in the reconstructed phase area of the ideal noise free hologram. Substituting these values in Equation.14 gives the standard deviation in phase error to be

$\sigma_{\theta_{e r r}}=\frac{\sigma_{n} f}{\sqrt{2} A}=\frac{\sqrt{\alpha \mu} f}{\sqrt{2} \alpha A}=\frac{\sqrt{\mu} f}{\sqrt{2} \alpha A}$

This shows that as the hologram is amplified by increasing $\alpha$, the standard deviation in the phase error decreases by a factor $\sqrt{\alpha}$. The filter factor $f$ is a constant for the system. It should be noted here that the amplification factor is applied to the holographic signal before the recording is made. This can be increased by increasing the power of the illuminating beams or by increasing the exposure time of the camera. It should also be noted that amplification by increasing the gain of the CCD only increases the sensitivity of the photo-detectors but does not decrease the photon shot noise.

To verify the theoretical model, we numerically introduce Poisson distributed noise into the hologram in order to simulate the presence of shot noise in our capture and then reconstruct using the convolutional approach. Poisson noise 
is signal dependent and to generate different noises, the mean value of the signal is varied by multiplying with a factor $\alpha$. Each pixel of the scaled hologram is treated as the mean of a Poisson distributed random variable and is replaced by a integer randomly selected from a generated distribution. There are various algorithms for generating Poisson distributed variables ${ }^{21}$.

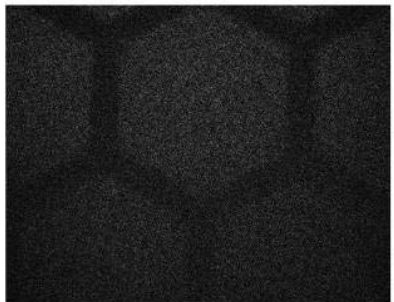

(a) Shot noise

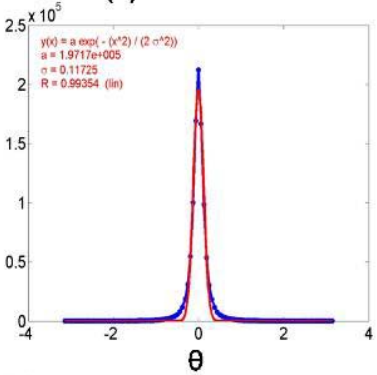

(c) Histogram of error in phase

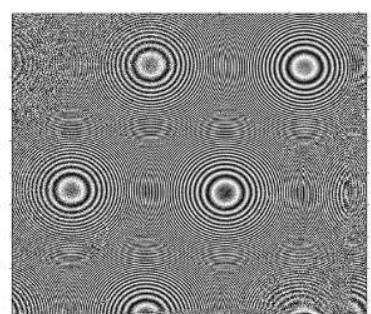

(b) Phase of reconstruction

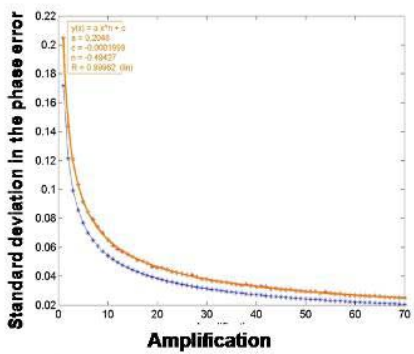

d) Phase error vs Amplification
Fig. 4 (a) Computer generated Poisson noise in the hologram (b) Noisy phase reconstruction (c) Histogram of the phase error with a least squares fit to a non-normalized Gaussian curve and (d) Standard deviation of the phase error vs. the amplification (a ) of the hologram. 'blue' curve is calculated using Equation 13 and the red curve is calculated by using computer generated Poisson noises for each value of the amplification factor (a ).

We use the imnoise function in Matlab to generate Poisson noises for all the holograms with different mean values. These holograms are reconstructed and the noisy phase values are calculated. A histogram of the phase error is calculated and the standard deviation is calculated by least squares fitting a non-normalized Gaussian to the histogram of the phase error. This value is plotted against the value given by Equation 13. Fig. 4(a) shows the shot noise in the hologram. Fig. 4(b) the phase reconstruction, Fig. 4(c) the histogram of the phase error with least squared fit of a Gaussian curve and Fig. 4(d) shows the standard deviation in the phase error for amplification factor $(\alpha)$ from 1 to 125 along with the predicted value from Equation 15.

As seen in Fig. 4(d), the standard deviation in the phase error follows a $\sqrt{\alpha}$ curve closely. Fig. 4(d) also shows the least squares fit (red line) to a function of the form $y=a \cdot x^{n}+c$ which gives the value of $n=-0.49$ and fit parameter $R=0.99$, confirming the theoretical inverse square root dependence. The same dependence was observed in experimental studies by the authors in ${ }^{12}$. Even though the simulation results and the predicted values follow the same $\sqrt{N}$ dependence, there is a difference in between the curves. We believe that this is due to the following assumptions. In the case of an appreciable modulation of the amplitude in the reconstruction plane, uniform noise in the hologram plane also becomes complex and Gaussian in the reconstruction plane. However in that case the phase error cannot be described by the equations here. The model used here relies on noise being added to a fixed amplitude phasor which is not the case when there is modulation in the amplitude of the signal. In our case there is non-uniformity (standard deviation to mean ratio of 0.21 ) in the amplitude due to various sources like spurious reflections, imperfections in optics, microscopic dust particles and the sample itself being not purely transparent.

Another assumption is one with the nature of the noise due to the Poisson process. It is signal dependent as can be seen in Fig. 4(a). Thus the assumption that the noise is completely random is not strictly true and thirdly our curve fitting is based on an approximation of Equation 11 under the assumption that $A>>\sigma_{R}$. For low image signal intensities, this assumption is not a strong one as the standard deviation of the Poisson noise is comparable to the signal.

\section{Conclusion}

We have quantified the influence of the additive noise on the phase measurement error in the hologram plane in digital holographic microscopy under the assumption that the object is highly transmissive in nature. We have shown that in general, the noise in the output plane of a Digital holographic system has Gaussian characteristics. This happens due to both the Fourier filtering as well as the numerical propagation of the complex wavefield. We have described the relationship between standard deviation in the error in the camera plane to the standard deviation in the error in the reconstructed phase in the case of Fresnel offaxis geometry. Shot noise which has Poisson statistics is a fundamental noise source for holographic imaging. According to our simple model, the phase error can be decreased linearly by amplifying the image by either increasing the exposure time or by adding multiple exposures. The reduction in error happens because the amplitude of the reconstructed image increases relative to the perturbation by the noise phasors in a $\sqrt{\alpha}$ fashion where $\alpha$ is the amplification of the holographic signal. We have studied both Gaussian and Poisson distributed noises in the camera plane and have verified the validity of our model using numerically generated noise.

\section{Acknowledgements}

The research leading to these results has received funding from the European community's seventh framework programme FP7/2007-2013 under grant agreement no. 216105 and also Science Foundation Ireland under the National Development Plan.

\section{References}

1. D. Carl, B. Kemper, G. Wernicke, G. von Bally (2004) Parameter-optimized digital holographic microscope for high-resolution living-cell analysis. Appl Opt. 43:6536-6544

2. B. Kemper, G. von Bally (2008) Digital holographic 
microscopy for live cell applications and technical inspection. Appl Opt. 47:A52-A61

3. P. Marquet, B. Rappaz, P. Magistretti, E. Cuche, Y. Emery, T. Colomb, C. Depeursinge (2005) Digital holographic microscopy: a noninvasive contrast imaging technique allowing quantitative visualization of living cells with subwavelength axial accuracy. $O p t$ Lett. 30:468-470

4. G. Pedrini, P. Fröning, H. Tiziani, F. Mendoza Santoyo (1999) Shape measurement of microscopic structures using digital holograms. Opt Comm. 164:257-268

5. J. Kühn, F. Charrière, T. Colomb, E. Cuche, F. Montfort, Y. Emery, P. Marquet, C. Depeursinge (2008) Axial sub-nanometer accuracy in digital holographic microscopy. Meas Sci Tech. 19:074,007

6. T. Colomb, J. Kühn, F. Charrière, C. Depeursinge, P. Marquet, N. Aspert (2006) Total aberrations compensation in digital holographic microscopy with a reference conjugated hologram. Opt Exp. 14:43004306

7. L. Miccio, D. Alfieri, S. Grilli, P. Ferraro, A. Finizio, L. De Petrocellis, S. Nicola (2007) Direct full compensation of the aberrations in quantitative phase microscopy of thin objects by a single digital hologram. Appl Phys Lett. 90:041,104

8. W. Davenport, W. Root (1958) An introduction to the theory of random signals and noise. McGraw-Hill New York.

9. M. Gross, M. Atlan (2007) Digital holography with ultimate sensitivity. Opt Lett. 32:909-911

10. M. Yamamoto, H. Yamamoto, Y. Hayasaki (2009) Photon-counting digital holography under ultraweak illumination. Opt Lett. 34:1081-1083

11. F. Charrière, T. Colomb, F. Montfort, E. Cuche, P. Marquet, C. Depeursinge (2006) Shot-noise influence on the reconstructed phase image signal-to-noise ratio in digital holographic microscopy. Appl Opt. 45:76677673

12. F. Charrière, B. Rappaz, J. Kühn, T. Colomb, P. Marquet, C. Depeursinge (2007) Influence of shot noise on phase measurement accuracy in digital holographic microscopy. Opt Express. 15:8818-8831

13. J. Stone (2004) Independent Component Analysis: A Tutorial Introduction. The MIT Press, Cambridge, Massachusetts.

14. T. Colomb, F. Montfort, C. Depeursinge (2008) Small Reconstruction Distance in convolution formalism. Digital Holography and Three-Dimensional Imaging.

15. BM. Hennelly, JT. Sheridan (2005) Generalizing, optimizing, and inventing numerical algorithms for the fractional Fourier, Fresnel, and linear canonical transforms. J Opt Soc Am A. 22:917-927

16. J. Goodman (2007) Speckle phenomena in optics: theory and applications. Roberts \& Co.

17. D. Middleton (1960) An introduction to statistical communication theory. McGraw-Hill New York.

18. H. Gudbjartsson, S. Patz (1995) The Rician distribution of noisy MRI data. Mag Res Med. 34:910914

19. B. Lathi (1995) Modern digital and analog communication systems. Oxford University Press, Inc. New York, NY, USA.

20. SS. Jiang, AA. Sawchuk (1986) Noise updating repeated wiener filter and other adaptive noise smoothing filters using local image statistics. Appl Opt. 25:2326-2337

21. J. Ahrens, U. Dieter (1974) Computer methods for sampling from gamma, beta, poisson and bionomial distributions. Computing. 12:223-246 